\title{
Effect of hyperbaric oxygen on lipid peroxidation and visual development in neonatal rats with hypoxia-ischemia brain damage
}

\author{
JING CHEN $^{1,2^{*}}$, YAN-HUI CHEN ${ }^{1 *}$, HONG-YAN LV ${ }^{1}$ and LI-TING CHEN ${ }^{1}$ \\ ${ }^{1}$ Department of Pediatrics, Union Hospital of Fujian Medical University, Fuzhou, Fujian 350001; \\ ${ }^{2}$ Department of Child Health, Xiamen Maternal and Child Health Care Hospital, Xiamen, Fujian 361003, P.R. China
}

Received January 14, 2016; Accepted April 19, 2016

DOI: 10.3892/br.2016.673

\begin{abstract}
The aim of the present study was to investigate the effect of hyperbaric oxygen (HBO) on lipid peroxidation and visual development in a neonatal rat model of hypoxic-ischemic brain damage (HIBD). The rat models of HIBD were established by delayed uterus dissection and were divided randomly into two groups (10 rats each): HIBD and HBO-treated HIBD (HIBD+HBO) group. Another 20 rats that underwent sham-surgery were also divided randomly into the HBO-treated and control groups. The rats that underwent HBO treatment received $\mathrm{HBO}(0.02 \mathrm{MPa}, 1 \mathrm{~h} /$ day $) 24 \mathrm{~h}$ after the surgery and this continued for 14 days. When rats were 4 weeks old, their flash visual evoked potentials (F-VEPs) were monitored and the ultrastructures of the hippocampus were observed under transmission electron microscope. The levels of superoxide dismutase (SOD) and malonyldialdehyde (MDA) in the brain tissue homogenate were detected by xanthine oxidase and the thiobarbituric acid colorimetric method. Compared with the control group, the ultrastructures of the pyramidal neurons in the hippocampal CA3 area were distorted, the latencies of F-VEPs were prolonged $(\mathrm{P}<0.01)$ and the SOD activities were lower while the MDA levels were higher $(\mathrm{P}<0.01)$ in the HIBD group. No significant differences in ultrastructure, the latency of F-VEPs or SOD/MDA levels were identified between the HBO-treated HIBD group and the normal control group $(\mathrm{P}>0.05)$. HBO enhances antioxidant capacity and reduces the ultrastructural damage induced by
\end{abstract}

Correspondence to: Professor Yan-Hui Chen, Department of Pediatrics, Union Hospital of Fujian Medical University, 29 Xinquan Road, Fuzhou, Fujian 350001, P.R. China

E-mail: yanhui_0655@126.com

${ }^{*}$ Contributed equally

Abbreviations: HBO, hyperbaric oxygen; HIBD, hypoxic-ischemic brain damage; SOD, superoxide dismutase; MDA, malondialdehyde; F-VEP, flash visual evoked potential

Key words: hyperbaric oxygen, hypoxic-ischemic brain damage, lipid peroxidation, flash visual evoked potential, rat hypoxic-ischemia, which may improve synaptic reconstruction and alleviate immature brain damage to promote the habilitation of brain function.

\section{Introduction}

Hypoxic-ischemic brain damage (HIBD) occurs at a rate of 3-5/1,000 full-term live births in developed countries and $\leq 10$-fold higher in developing countries (1). Following hypoxia-ischemia (HI), $45 \%$ of newborns succumb or have permanent cognitive impairments, potentially including cerebral palsy, and there are currently no effective therapies (2).

Hyperbaric oxygen (HBO) therapy has been identified as a clinical therapy for HIBD for a number of years. Animal experiments and clinical trials have shown that $\mathrm{HBO}$ performed within $6 \mathrm{~h}$ after HIBD may achieve favorable outcomes and promote the long-term neurological recovery (3-6). In addition, numerous studies in China have demonstrated that $\mathrm{HBO}$ can reduce the disability and mortality of HIBD (7). However, there are concerns regarding the safety and validity of $\mathrm{HBO}$ treatment due to oxygen toxicity, which may increase the infarct area (3). A previous study demonstrated an increase in lipid and protein oxidation products in the lung tissues of rats accompanied by increased antioxidant enzyme activities when $\mathrm{HBO}$ was continued for $>20$ sessions (8). In addition to the lung being the entering site of hyperoxic injuries, CNS is mainly accepted as another important target for oxygen exposure in toxic amounts (9). However, whether HBO will exacerbate lipid peroxidation causing secondary injury to the brain or lead to neonatal pulmonary and retinal hypoplasia (10) remains to be elucidated. The aim of the present study was to detect the effect of HBO on superoxide dismutase (SOD) and malondialdehyde (MDA) from brain homogenate and flash visual evoked potential (F-VEP) in newborn rats with HIBD through the establishment of the HIBD newborn rat model, to explore the influence of $\mathrm{HBO}$ on brain lipid peroxidation and visual development, and to provide experimental evidence for the clinical application of HBO.

\section{Materials and methods}

Preparation for animal model and grouping. Intrauterine HIBD animal models were established according to the method of 'delayed dissect uterus' by Bjelke et al (11). 
Sprague-Dawley rats on gestational day 21, weighing 300-350 g, were selected. To perform uterine cornual artery ligation, the rats were anesthetized with ether. Rats have bicornuate uteri and the dissected uteri from the side that the cornual arteries were ligated was immersed in water at $37^{\circ} \mathrm{C}$. A cesarean section was performed 15 min later. Neonatal rats that had reached the level of severe brain damage according to HIBD brain damage evaluation standards (12) were the HIBD models (the HIBD group). The neonatal rats from the side of the uterus in which the side cornual arteries were not ligated were the control group. All rats were fed by female rats who had a spontaneous delivery one day before. Rats with HIBD showed convulsion-like complications, and the pathological changes conform to that of typical HIBD (13). Referring to a method established by Whishaw et al (13), a pathological score was assigned to the brain tissue, and the level of HIBD brain damage pathology may correspond to its clinical manifestation. According to the weight and degree of brain damage, the model rats were randomly divided into the HIBD and HIBD with HBO treatment (HIBD+HBO) groups. The rats in the control group without HIBD were also divided into HBO-treated group (HBO group) and the control group randomly. Finally, there were 4 groups with 10 neonatal rats each: The HIBD, HIBD+HBO, HBO and control groups.

Ethical approval. The study protocol was approved by the Ethics Committee of the Union Hospital of Fujian Medical University (Fujian, China).

$H B O$ exposure. In accordance with previous protocols $(14,15)$, the rats in the $\mathrm{HBO}$ group and HIBD+HBO group were placed into a hyperbaric chamber (model YLC 0.5/1 A baby hyperbaric chamber; China State Shipbuilding Corporation 701, Beijing, China) $24 \mathrm{~h}$ after surgery and were administered HBO treatment (100\% oxygen under $0.02 \mathrm{MPa}$ for $30 \mathrm{~min}$ ). The pressure gradually reached a maximum of $0.02 \mathrm{MPa}$ in $15 \mathrm{~min}$, which was maintain for another $30 \mathrm{~min}$, and gradually reduced to normal. The total procedure lasted $1 \mathrm{~h}$ and the treatment continued daily for 14 consecutive days.

F-VEP monitoring. Immediately following 14 days of HBO treatment, all the animals were anesthetized with an equivalence combination ( $3 \mathrm{ml} / \mathrm{kg}$ intraperitoneally) of $20 \%$ urethane and $10 \%$ chloral hydrate. The F-VEPs were monitored by neural electrophysiological instrumentation (NDI-200F; Shanghai Navy Medical Research Institute, Shanghai, China). The frequency of flash stimulation was $3 \mathrm{~Hz}$ and the electronic signal was amplified with a filter setting of 1-100 Hz at a gain of 200 and the sensitivity was $20 \mu \mathrm{V}$. The P1 latencies of F-VEPs were measured as previously described (16).

Tissue preparation and ultrastructural changes. When the rats were at 4 postnatal weeks, 3 rats were randomly selected from each group. Following rapid decapitation, the hippocampi were quickly removed (tissue size, $\sim 1 \mathrm{x} 1 \mathrm{x} 1 \mathrm{~mm} ; 2$ samples each in the CA3 area). Subsequently, the specimens underwent a procedure of pre-fixation in $3 \%$ glutaraldehyde and $1.5 \%$ paraformaldehyde, post-fixation in $1 \%$ osmic acid and $1.5 \%$ potassium ferrocyanide, subsequently dehydrated with
Table I. P1 latencies of the flash visual evoked potential of rats in the different groups.

\begin{tabular}{lc}
\hline Groups & Latency of P1, msec \\
\hline HBO & $42.94 \pm 4.87^{\mathrm{a}}$ \\
Control & $48.67 \pm 4.15$ \\
HIBD+HBO & $50.24 \pm 4.46^{\mathrm{b}}$ \\
HIBD & $58.60 \pm 5.12^{\mathrm{c}}$ \\
\hline
\end{tabular}

Compared with the control group, ${ }^{\mathrm{a}} \mathrm{P}<0.05$; with the HIBD group, ${ }^{b} \mathrm{P}<0.01$; and with the control group, ${ }^{\mathrm{c}} \mathrm{P}<0.01$. Data are mean \pm standard deviation $(n=10)$. HBO, hyperbaric oxygen; HIBD, hypoxic-ischemic brain damage.

ethanol and acetone, and finally embedded in Epoxy resin 618. The ultrathin sections were observed under the transmission electron microscope (Hitachi Hu-12A type; Hitachi, Ltd., Tokyo, Japan) following counterstaining by uranyl acetate and lead citrate.

SOD activity and MDA level. SOD activities or MDA levels in the hippocampal homogenates of rats were separately assayed by the method of xanthine oxidase or thiobarbituric acid, according to the protocols of the SOD and MDA kits [Nanjing Jianchen Biological Engineering Research Institute, Nanjing, China (cat. nos. 20080714 and 20080714, respectively)].

Statistical analysis. Analysis of variance was performed with SPSS 11.0 statistical software (SPSS, Inc., Chicago, IL, USA). The results are expressed as mean \pm standard deviation. $\mathrm{P}<0.05$ was considered to indicate a statistically significant difference.

\section{Results}

P1 latencies of F-VEPs. In the F-VEP test, the rats (aged 28 days) in the HBO group had shorter P1 latencies compared to those in the control group $(\mathrm{P}<0.05)$, while in the HIBD group the rats had prolonged P1 latencies compared with those in the control group $(\mathrm{P}<0.01)$. The $\mathrm{P} 1$ latencies of rats in the HIBD+HBO group were shorter than those in the HIBD group $(\mathrm{P}<0.01)$. No significant differences were identified in the $\mathrm{P} 1$ latencies of rats between the HIBD+HBO and control groups $(\mathrm{P}>0.05)$ (Table I).

Ultrastructure changes in CA3 pyramidal neurons. Under the transmission electron microscope, the pyramidal neurons of the hippocampal CA3 area in the HBO and control groups exhibited a clear nuclear membrane, evident nucleolus, rich organelles and complete synaptic structure. In contrast to the control group, the rats in the HIBD group showed distorted nuclei with partial disappearance of the nuclear membrane, vague mitochondria cristae, decreasing presynaptic vesicles, thin postsynaptic density, unevenly widened synaptic space, and disruption of whole synaptic structures due to presynaptic membrane damage. No differences in neural cells and synaptic structure were identified between the HIBD+HBO and control groups (Figs. 1-4). 
Table II. Changes of SOD activities and MDA levels in the rat brain between the different treatment groups.

\begin{tabular}{lcc}
\hline Groups & SOD, U/mg protein & MDA, U/mg protein \\
\hline HBO & $117.23 \pm 10.55^{\mathrm{a}}$ & $10.31 \pm 2.11^{\mathrm{a}}$ \\
Control & $94.15 \pm 8.93$ & $16.65 \pm 3.12$ \\
HIBD+HBO & $88.69 \pm 7.25^{\mathrm{b}}$ & $17.02 \pm 3.71^{\mathrm{b}}$ \\
HIBD & $73.67 \pm 10.26^{\mathrm{c}}$ & $27.16 \pm 4.66^{\mathrm{c}}$ \\
\hline
\end{tabular}

Compared with the control group, ${ }^{a} \mathrm{P}<0.05$; with the HIBD group, ${ }^{\mathrm{b}} \mathrm{P}<0.01$; and with the control group, ${ }^{\mathrm{c}} \mathrm{P}<0.01$. SOD, superoxide dismutase; MDA, malondialdehyde; HBO, hyperbaric oxygen; HIBD, hypoxic-ischemic brain damage. Data are mean \pm standard deviation.

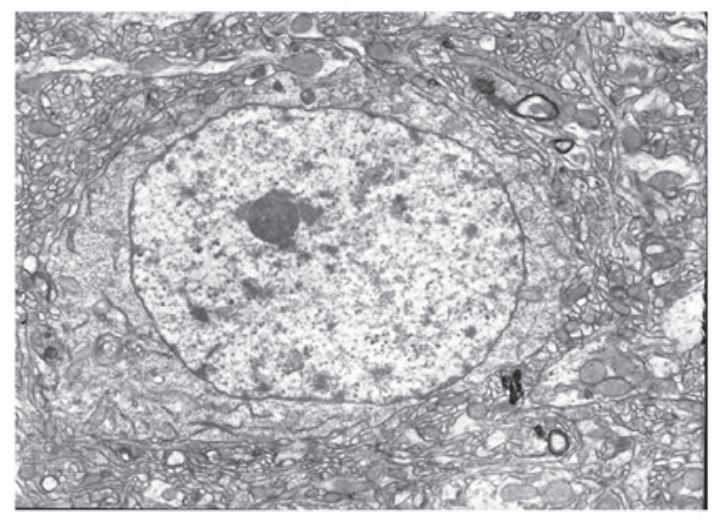

Figure 1. Ultrastructure of the neuron in the hippocampus CA3 of rats in the hypoxic-ischemic brain damage + hyperbaric oxygen group (transmission electron microscope magnification, $\mathrm{x} 4,800$ ).

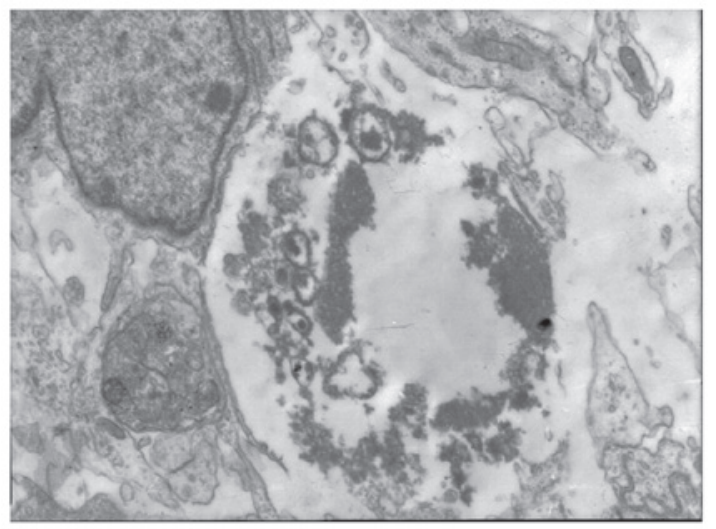

Figure 2. Ultrastructure of the neuron in the hippocampus CA3 of rats in the hypoxic-ischemic brain damage group (transmission electron microscope magnification, $\mathrm{x} 9,000)$.

Changes of SOD activities and MDA levels. The results showed that the SOD activities in the rat brain from the HIBD group were lower $(\mathrm{P}<0.01)$ and the levels of MDA were higher $(\mathrm{P}<0.01)$ compared to those from the control group. HBO intervention can increase the SOD activity and decrease MDA levels in the brain tissue of rats from the HIBD group $(\mathrm{P}<0.01)$ and the control group $(\mathrm{P}<0.05)$. No significant differences were identified in the changes of SOD activities and MDA

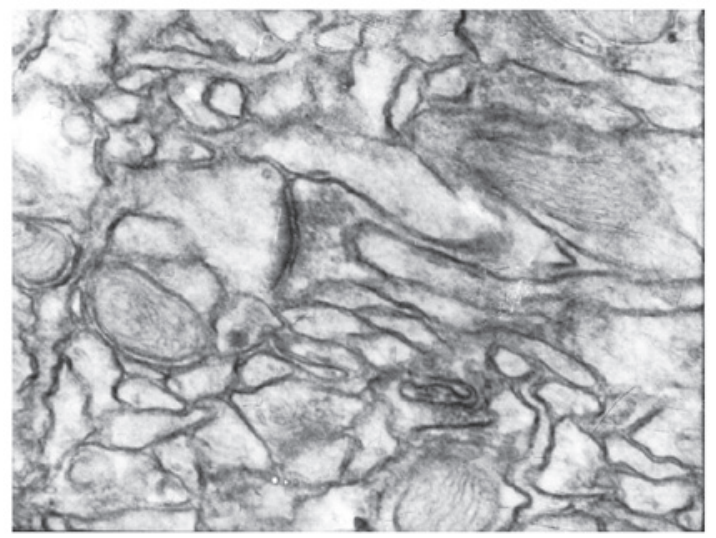

Figure 3. Ultrastructure of synapse in the hippocampus CA3 of rats in the hypoxic-ischemic brain damage + hyperbaric oxygen group (transmission electron microscope magnification, $\mathrm{x} 30,000)$.

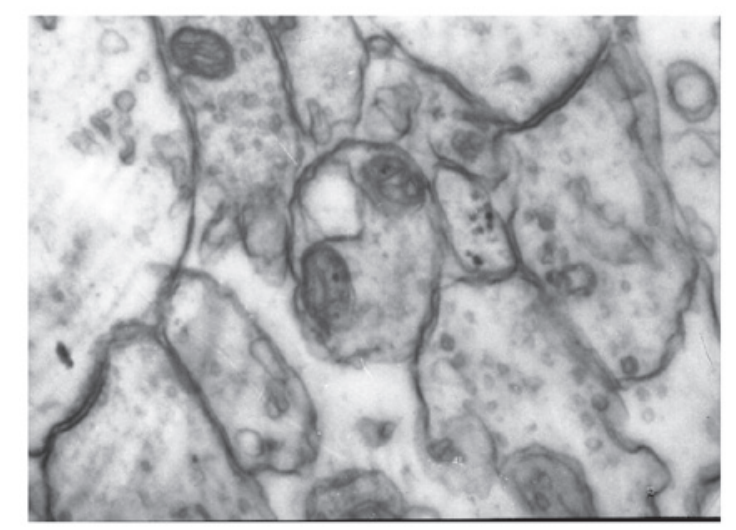

Figure 4. Ultrastructure of the synapse in the hippocampus CA3 of rats in the hypoxic-ischemic brain damage group (transmission electron microscope magnification, $\mathrm{x} 30,000)$.

levels between the HBO-treated HIBD group and the control group (Table II).

\section{Discussion}

Compared with the hypoxic ischemia model caused by ligating the side carotid artery postnatally, intrauterine HIBD animal models better replicate the common syndrome of fetal distress, which is more similar to clinical conditions. The intrauterine HIBD animal models were established by ligating the side cornual artery and delayed cesarean section (11). The neonatal rats in the control group came from the other side of the uterus where the cornual artery was not ligated. The rats of the experimental group and the control group were from the same mother, which may reduce any genetic influence. The rats had moderate or severe brain damage in HIBD and may have had sequelae such as mental retardation and cerebral palsy. This is why only rats with moderate or severe brain damage were used as HIBD models in the present study.

HBO was widely adopted as a clinical therapy for anoxia disease as it can increase the blood oxygen content and partial pressure of oxygen in arteries, enhance the utilization of oxygen in plasma and tissue fluids, and improve the diffusion of oxygen through tissue fluids from the capillary to the cells. 
However, whether the application of HBO therapy in neonates was effective or if it would impede the development of pulmonary and retinal structures in immature individuals remains to be elucidated.

F-VEPs are bioelectrical activity caused by the visual stimulations along different sections of optic and visual pathway, which were used to evaluate neural pathways from the optic chiasm level to part under ventricle to the visual cortex level, and it should be consistent with vulnerable sections of classic HIBD. The P1 waves of F-VEPs are stable composite waves based on positive waves, of which the latency is an important index in F-VEP tests. The P1 latency is associated with myelination of nerve fibers and synaptic transmission efficiency, and is independent from anesthetics or sedatives. Shorter P1 latencies indicate more perfect neurodevelopment and a more complete visual path (17). In the present study, rats in the HIBD group showed longer P1 latencies compared with the control group prior to HBO treatment, which implied decreased nerve fiber myelination and synaptic transmission efficiency in neonatal hypoxic-ischemic brain. After continuous HBO treatment for 14 days ( $1 \mathrm{~h} /$ day), the P1 latencies of the rats at 4 weeks postnatal in the HIBD group were evidently shorter compared to those without HBO therapy, and were similar to those of the rats in the control group. The result suggested that HBO treatment cannot impede normal visual development.

Synapses are specialized structures conveying information between neurons in the nervous system, and synaptic knobs contain numerous vesicles, which are closely associated with neurotransmitter release (18). Postsynaptic densities are the main components of postsynaptic membrane, of which the alteration in thickness is one of the most important morphological bases for normal synaptic function (19). Synaptic space contains a lot of lytic enzyme which can decompose the neurotransmitter. The wider the synaptic space is, the longer the time that neurotransmitter conduction takes; more were degraded during the conduction period, thus causing a reduction of the abilities of nerve conduction and learning and memory. In the present study, transmission electron microscopy showed that there were evident morphological changes in the hippocampal CA3 neurons of rats in the HIBD group, of which the nuclear membranes disappeared in part, the mitochondria cristae were vague, the number of presynaptic vesicles decreased, the postsynaptic density became thin, and the synaptic clefts were unevenly widened; whole synapses were also destroyed. However, in the HBO-treated HIBD group, no significant differences were identified in CA3 synaptic ultrastructures compared with that in the control group under the transmission electron microscope. The latency of F-VEP of rats in HIBD following the HBO intervention was shorter than that in the control group, which suggested that early HBO intervention could maintain a relatively normal synaptic structure and their functions by reducing synaptic ultrastructural damage caused by hypoxia ischemia and promoting restoration from injuries.

Lipid peroxidation is considered an important reason for immature brain injury, pulmonary hypoplasia and visual damage in oxygen therapy $(3,20,21)$. MDA is an end product in lipid peroxidation which can reflect the extent of lipid peroxidation. SOD is a main antioxidant enzyme in organisms that has an important role in removing extra free radicals. The present results showed that the SOD content of rats in the HIBD group was markedly lower while the MDA content was significantly higher than those in the control group, which implied that HI could reduce the antioxidant abilities and promote lipid peroxidation. Following the HBO intervention, the rats with HIBD showed significantly increased SOD activity and decreased MDA content, which suggested that a HBO intervention could markedly improve the removal of oxygen free radicals and resist lipid peroxidation. The study showed that HBO could enhance antioxidant capacity and decrease the ultrastructural damage induced by hypoxic-ischemia, and facilitate synaptic reconstruction and alleviate injury on immature brain thus promoting the rehabilitation of brain function. These results support that intervention programs and a pressure of $\mathrm{HBO}$ (0.02 MPa once a day for $1 \mathrm{~h} ; 14$ days total) do not increase oxidative damage of the central nervous system of the HIBD rats. Instead, antioxidant injury is conducive to a protective effect on the brain. Future investigations are required to elaborate upon the mechanism of $\mathrm{HBO}$ treatment on the immature brain and to identify the association between the therapeutic effect of $\mathrm{HBO}$ treatment and the pressure or time and duration of the HBO intervention.

\section{Acknowledgements}

The authors thank Dr Ming-Qi Qiu of Union Hospital of Fujian Medical University for her helpful advice. The present study was supported by grants from the Advanced Research Fund of Fujian Medical University for Professors (no. JS07008) and the Key Program of Scientific Research of Fujian Medical University (no. 2009D064).

\section{References}

1. Sanders RD, Manning HJ, Robertson NJ, Ma D, Edwards AD, Hagberg $\mathrm{H}$ and Maze M: Preconditioning and postinsult therapies for perinatal hypoxic-ischemic injury at term. Anesthesiology 113: 233-249, 2010.

2. Chen W, Ostrowski RP, Obenaus A and Zhang JH: Prodeath or prosurvival: Two facets of hypoxia inducible factor-1 in perinatal brain injury. Exp Neurol 216: 7-15, 2009.

3. Badr AE, Yin W, Mychaskiw G and Zhang JH: Dual effect of HBO on cerebral infarction in MCAO rats. Am J Physiol Regul Integr Comp Physiol 280: R766-R770, 2001.

4. Calvert JW, Zhou C, Nanda A and Zhang JH: Effect of hyperbaric oxygen on apoptosis in neonatal hypoxia-ischemia rat model. J Appl Physiol 1985 95: 2072-2080, 2003.

5. Veltkamp R, Siebing DA, Heiland S, Schoenffeldt-Varas P, Veltkamp C, Schwaninger M and Schwab S: Hyperbaric oxygen induces rapid protection against focal cerebral ischemia. Brain Res 1037: 134-138, 2005.

6. Nemoto EM and Betterman K: Basic physiology of hyperbaric oxygen in brain. Neurol Res 29: 116-126, 2007.

7. Yin X, Meng F, Wang Y, Wei W, Li A, Chai Y and Feng Z: Effect of hyperbaric oxygen on neurological recovery of neonatal rats following hypoxic-ischemic brain damage and its underlying mechanism. Int J Clin Exp Pathol 6: 66-75, 2013.

8. Simsek K, Ay H, Topal T, Ozler M, Uysal B, Ucar E, Acikel CH, Yesilyurt O, Korkmaz A, Oter S, et al: Long-term exposure to repetitive hyperbaric oxygen results in cumulative oxidative stress in rat lung tissue. Inhal Toxicol 23: 166-172, 2011.

9. Chavko M and Harabin AL: Regional lipid peroxidation and protein oxidation in rat brain after hyperbaric oxygen exposure. Free Radic Biol Med 20: 973-978, 1996.

10. Liu MN, Zhuang SQ, Zhang HY, Qin ZY and Li XY: Long-term effects of early hyperbaric oxygen therapy on neonatal rats with hypoxic-ischemic brain damage. Zhongguo Dang Dai Er Ke Za Zhi 8: 216-220, 2006 (In Chinese). 
11. Bjelke B,Andersson K, Ogren SO and Bolme P: Asphyctic lesion: Proliferation of tyrosine hydroxylase-immunoreactive nerve cell bodies in the rat substantia nigra and functional changes in dopamine neurotransmission. Brain Res 543: 1-9, 1991.

12. Wang R, Xu J, Xie J, Kang Z, Sun X, Chen N, Liu L and $\mathrm{Xu} \mathrm{J}$ : Hyperbaric oxygen preconditioning promotes survival of retinal ganglion cells in a rat model of optic nerve crush. J Neurotrauma 27: 763-770, 2010.

13. Whishaw IQ, Rod MR and Auer RN: Behavioral deficits revealed by multiple tests in rats with ischemic damage limited to half of the CA1 sector of the hippocampus. Brain Res Bull 34: 283-289, 1994.

14. Thiel G: Synapsin I, synapsin II, and synaptophysin: Marker proteins of synaptic vesicles. Brain Pathol 3: 87-95, 1993.

15. Zhu M, Lu M, Li QJ, Zhang Z, Wu ZZ, Li J, Qian L, Xu Y and Wang ZY: Hyperbaric oxygen suppresses hypoxic-ischemic brain damage in newborn rats. J Child Neurol 30: 75-82, 2015.

16. Feng Y, Zhang Z, Li Q, Li W, Xu J and Cao H: Hyperbaric oxygen preconditioning protects lung against hyperoxic acute lung injury in rats via heme oxygenase-1 induction. Biochem Biophys Res Commun 456: 549-554, 2015.
17. Gasier HG and Fothergill DM: Oxidative stress, antioxidant defenses and nitric oxide production following hyperoxic exposures. Undersea Hyperb Med 40: 125-134, 2013.

18. Bona E, Johansson BB and Hagberg H: Sensorimotor function and neuropathology five to six weeks after hypoxia-ischemia in seven-day-old rats. Pediatr Res 42: 678-683, 1997.

19. Chen HJ, Zhang ZD and Ju JD: Morphology and quantitative pathological score of brain in neonatal rats with hypoxic-ischemic brain damage. Shanghai Med J 23: 682-684, 2000.

20. Calvert JW, Yin W, Patel M, Badr A, Mychaskiw G, Parent AD and Zhang JH: Hyperbaric oxygenation prevented brain injury induced by hypoxia-ischemia in a neonatal rat model. Brain Res 951: 1-8, 2002

21. Xue M, Chen JQ and Chen R: Research of visual evoked potential in neonatal rats with hypoxic-ischemic brain damage. Acta Univ Medicinalis Nanjing 21: 295-298, 2001. 